\title{
SOME THIN LIE ALGEBRAS RELATED TO ALBERT-FRANK ALGEBRAS AND ALGEBRAS OF MAXIMAL CLASS
}

\author{
A. CARANTI and S. MATTAREI \\ Dedicated to M. F. (Mike) Newman on the occasion of his 65th birthday
}

(Received 18 December 1998; revised 26 May 1999)

Communicated by E. A. O'Brien

\begin{abstract}
We investigate a class of infinite-dimensional, modular, graded Lie algebra in which the homogeneous components have dimension at most two. A subclass of these algebras can be obtained via a twisted loop algebra construction from certain finite-dimensional, simple Lie algebras of Albert-Frank type.

Another subclass of these algebras is strictly related to certain graded Lie algebras of maximal class, and exhibits a wide range of behaviours.

1991 Mathematics subject classification (Amer. Math. Soc.): primary 17B50, 17B70, 17B65, 17B68, $17 \mathrm{~B} 56$.

Keywords and phrases: modular Lie algebras, graded Lie algebras, infinite-dimensional Lie algebras, cohomology of Lie algebras.
\end{abstract}

\section{Introduction}

The program of classification of pro-p-groups of finite coclass proposed in [19] has been completed in [18] and [25]. It is natural to investigate families of pro-p-groups that extend the family of pro- $p$-groups of finite coclass. One such family is that of groups of finite width [20], in which the order of the lower central factors is bounded.

In [4] a subfamily of such groups has been introduced, that of thin groups. These are groups whose lower central factors are elementary abelian, of rank at most 2 ,

Partially supported by MURST, Italy. First author partially supported by EU via HCM contract CHRXCT 93-0418 'Computational Group Theory'.

Authors are members of CNR-GNSAGA, Italy.

(C) 1999 Australian Mathematical Society 0263-6115/99 \$A2.00+0.00 
and satisfy a further covering property. This property can actually be read on the associated graded Lie ring (see (1) below), which in this case is a Lie algebra over a prime field. The approach to thin groups via the associated Lie algebra has been taken in [9], where the location of the second diamond in such a group was investigated. A lower central factor of rank two in a thin group or in its associated Lie algebra is called a diamond. There is of course a diamond at the top of a thin group. It follows from the theory of $p$-groups of maximal class (see for instance [13, III.14]) that in a thin group the second diamond occurs in class at most $p$. In [9] we proved that the associated Lie algebra has bounded dimension, except when the second diamond occurs in class 3, 5 or $p$. Now the Nottingham group is a thin group, in which the second diamond occurs in class $p$. The connection back from Lie algebras to groups has been completed by the results of [22].

In [5] we studied the graded Lie algebra associated to the Nottingham group with respect to its lower central series, and more generally Lie algebras in which the second diamond occurs in class which is a power of $p$, the characteristic of the underlying field.

The arguments of [9] are extended in [2] to show that there can be infinitedimensional thin algebras, besides the cases already mentioned, only if the second diamond occurs in class congruent to -1 modulo $p$. Here we begin to study these algebras. It turns out that in such an algebra the second diamond actually occurs in a class of the form $-1+2 q$, where $q$ is a power of the characteristic of the underlying field. It follows that the Lie algebras we study here have no counterpart in groups.

One can attach to the diamonds of these algebras a certain parameter, with values in the prime field plus infinity, which we call the type of the diamond. If all diamonds are of infinite type, the resulting algebras exhibit a close connection (Theorem 2 and Theorem 4) to some of the algebras of maximal class studied in [8] and [10]. It follows from the theory of the latter algebras that there is a wide variety of thin algebras of this type.

If the second diamond is of finite type, then we obtain that the algebra is uniquely determined (Theorem 5) by the value of $q$ as a (twisted) loop algebra of a certain finitedimensional simple algebra of Albert-Frank type [1]. (See also [3].) It is convenient to consider also the loop algebra of a certain central extension of this algebra. We prove that the latter loop algebra is finitely presented, and this in turn yields uniqueness for the corresponding thin algebra.

In the related paper [7] we will provide, among others, an explicit identification of this finite-dimensional simple Lie algebra with a non-graded Hamiltonian algebra. This follows implicitly from the classification of modular, simple Lie algebras over algebraically closed fields of characteristic greater than 7 (see [17]). However, we have not been able to find such an explicit identification over the ground field in the literature. 
Our methods are mostly based on direct computations. These rely in turn on evaluations of (sums of) binomial coefficients modulo a prime, exploiting Lucas' Theorem. The details are elementary, but the reason why we perform a particular calculation may appear obscure. We believe that there are deeper combinatorial reasons behind all this, probably related to the subtle problem of what exactly are the Lie words in positive characteristic [26], but this eludes us at this stage. In our calculations, we have been led by computational experiments with the Australian National University $p$-Quotient Program (ANU po, see [12]), as explained elsewhere [9].

Computational evidence also appears to show that there are thin algebras in this class containing diamonds of both finite and infinite type; we plan to come back to these algebras in another paper.

We are grateful to Claretta Carrara and Marina Avitabile for pointing out several errors in previous versions. We are grateful to the editor and the referees for several useful remarks and corrections.

\section{Notation}

We write $\mathbf{F}_{q}$ for the finite field with $q$ elements. We recall that we have

$$
\sum_{\alpha \in \mathbf{F}_{q}} \alpha^{t}= \begin{cases}-1 & \text { if } t \equiv 0 \quad(\bmod q-1) \\ 0 & \text { otherwise. }\end{cases}
$$

We write Kronecker's $\delta$ in the form

$$
\delta(a, b)= \begin{cases}1 & \text { if } a=b \\ 0 & \text { otherwise }\end{cases}
$$

On one occasion we have integers $a, b$ that are also considered as elements of a prime field $\mathbf{F}_{p}$. We then write

$$
\delta_{p}(a, b)= \begin{cases}1 & \text { if } a \equiv b \quad(\bmod p), \\ 0 & \text { otherwise }\end{cases}
$$

to avoid ambiguities.

We also use several times Lucas' Theorem [21], which states the following. Let $a$ and $b$ be two non-negative integers, and $p$ a prime. Write $a$ and $b$ in $p$-adic form,

$$
\begin{aligned}
& a=a_{0}+a_{1} p+\cdots+a_{n-1} p^{n-1}+a_{n} p^{n}, \\
& b=b_{0}+b_{1} p+\cdots+b_{n-1} p^{n-1}+b_{n} p^{n},
\end{aligned}
$$


that is, $0 \leq a_{i}, b_{i}<p$, for all $i$. Then we can compute the binomial coefficient modulo $p$ as

$$
\left(\begin{array}{l}
a \\
b
\end{array}\right) \equiv\left(\begin{array}{l}
a_{0} \\
b_{0}
\end{array}\right)\left(\begin{array}{l}
a_{1} \\
b_{1}
\end{array}\right) \cdots\left(\begin{array}{l}
a_{n-1} \\
b_{n-1}
\end{array}\right)\left(\begin{array}{l}
a_{n} \\
b_{n}
\end{array}\right) \quad(\bmod p) .
$$

We call $a_{0}$ the the first p-adic digit of $a$.

We use several times, without explicit mention, the standard expansion in a $\mathrm{Lie}$ algebra

$$
\left[v\left[y z^{\lambda}\right]\right]=\sum_{i=0}^{\lambda}(-1)^{i}\left(\begin{array}{l}
\lambda \\
i
\end{array}\right)\left[v z^{i} y z^{\lambda-i}\right] .
$$

Note that here and in the following our repeated commutators are left-normed, that is, $[x y z]=[[x y] z]$, and we use the notation

$$
\left[y z^{\lambda}\right]=[y \underbrace{z \cdots z}_{\lambda}] .
$$

We write $\langle u, v, \ldots\rangle$ for the subspace spanned by the vectors $u, v, \ldots$ in an appropriate vector space.

We use [14] and [27] as general references.

\section{Preliminaries}

Let

$$
L=\bigoplus_{i=1}^{\infty} L_{i}
$$

be a graded Lie algebra over a field $\mathbf{F}$ of characteristic $p>3$. We will say that $L$ is a thin algebra when $\operatorname{dim}\left(L_{1}\right)=2$, and the following covering property holds

$$
\text { for all } i \geq 1 \text {, and all } u \in L_{i}, u \neq 0 \text {, we have } L_{i+1}=\left[u L_{1}\right] \text {. }
$$

It foliows from (1) that $L$ is generated by elements of weight 1 , that is, $L_{i+1}=\left[L_{i} L_{1}\right]$, for $i \geq 1$. It also follows that $\operatorname{dim}\left(L_{i}\right) \leq 2$ for all $i$, so that a thin algebra is of finite width. We call a homogeneous component $L_{i}$ of dimension 2 a diamond. Therefore $L_{1}$ is a diamond. If there are no other diamonds, than $L$ is an algebra of maximal class (see [8]). By convention, we do not consider algebras of maximal class to be thin. If there are other diamonds, then we refer to the finite sequence of the homogeneous components of dimension 1 between two consecutive diamonds as a chain. 
Note that the same definition makes perfect sense even if the grading is not over the positive integers, but over a cyclic group. We will make use of this later.

When the grading is over the positive integers, it is convenient to take thin algebras $L$ to be infinite-dimensional, that is, $L_{i} \neq 0$ for all $i$. However most of what we do makes sense for algebras that have large enough class (see [8] for similar details).

Suppose $L$ is an infinite-dimensional thin algebra in which the second diamond occurs in class $k \equiv-1(\bmod p)$, and that we have

$$
C_{L_{1}}\left(L_{2}\right)=C_{L_{1}}\left(L_{3}\right)=\cdots=C_{L_{1}}\left(L_{k-2}\right)=\langle y\rangle .
$$

We call such an algebra a (-1)-algebra.

We refer to [6] for a proof of the fact that (2) is not restrictive in odd characteristic; see also [16] for the case of characteristic two.

Choose arbitrarily $x \in L_{1} \backslash\langle y\rangle$, so that $x$ and $y$ generate $L$. The element

$$
v=\left[y x^{k-2}\right]
$$

is thus nonzero, and has weight $k-1$. Note first that $k$ is odd. In fact, if $k=2(h+1)$, then

$$
0=\left[\left[y x^{h}\right]\left[y x^{h}\right]\right]=(-1)^{h}\left[y x^{2 h} y\right]=(-1)^{h}[v y],
$$

and there is no diamond in class $k$ after all.

Now write $h=(k-1) / 2 \equiv-1(\bmod p)$. We have

$$
0=\left[\left[y x^{h}\right]\left[y x^{h}\right]\right]=(-1)^{h-1}\left(\begin{array}{c}
h \\
h-1
\end{array}\right)[v y x]+(-1)^{h}[v x y],
$$

that is,

$$
[v x y]+[v y x]=0 .
$$

Take an element $v^{-1} \in L_{k-2}$ so that $\left[v^{-1} x\right]=v$. We have $\left[v^{-1} y\right]=0$, and thus we obtain

$$
0=\left[v^{-1}[y x y]\right]=-\left[v^{-1} x y y\right]+2\left[v^{-1} y x y\right]-\left[v^{-1} y y x\right]=-[v y y] .
$$

By the covering property, $L_{k+1}=\langle[v y x],[v y y]\rangle=\langle[v y x]\rangle$ is one-dimensional. Therefore, $[v x x]$ must be a multiple of $[v y x]$. From (3)

$$
[v, x+\alpha y, x+\alpha y]=[v x x]+\alpha([v x y]+[v y x])+\alpha^{2}[v y y]=[v x x] .
$$

If $[v x x]=0$, then we say the the second diamond is of infinite type. Note that this condition is independent of the choice of $x$. Otherwise, we say that the second diamond is of finite type. We refine these definitions later. 


\section{The first chain}

In this section we prove the following result, which is critical in the sequel.

PROPOSTTION 1. Let L be a (-1)-algebra over a field of characteristic $p>2$, with second diamond in weight $k \equiv-1(\bmod p)$. Since $k$ is odd, we can write

$$
k=-1+2 q n,
$$

where $q=p^{h}$ for some positive $h$, and $n \not \equiv 0(\bmod p)$. If $n>1$, then $L$ is finite-dimensional, of dimension at most $k+q+1$.

ProOF. Write $0 \neq v=\left[y x^{k-2}\right] \in L_{k-1}$. Recall that we have [vyy] $=0=$ $[v x y]+[v y x]$. Take $j<q-3$. We want to prove, proceeding by induction on $j$, that

$$
\left[v y x x^{j} y\right]=0 .
$$

We have $\left[y x^{j+1} y\right]=0$, so that

$$
\begin{aligned}
0 & =\left[\left[y x^{k-2}\right]\left[y x^{j+1} y\right]\right]=\left[\left[y x^{k-2}\right]\left[y x^{j+1}\right] y\right]-\left[y x^{k-2} y\left[y x^{j+1}\right]\right] \\
& =\left[y x^{k-2} y x x^{j} y\right]-(j+1)\left[y x^{k-2} x y x^{j} y\right]-(-1)^{j+1}\left[y x^{k-2} y x x^{j} y\right] \\
& =\left(j+2-(-1)^{j+1}\right)\left[y x^{k-2} y x x^{j} y\right] .
\end{aligned}
$$

This fails to show what we need when $j+1 \cong 0(\bmod p)$ (for odd $j$ ), and when $j+3 \equiv 0(\bmod p)($ for even $j)$.

In the latter case, write $j+3=\beta p^{t}$, where $\beta \neq 0(\bmod p)$. Since $j<q-3$, we have $p^{t}<q$, so that $j+p^{t}+1<q-3+q \leq 2 q n-3$. Therefore $\left[y x^{j+p^{\prime}+1} y\right]=0$, and we obtain

$$
\begin{aligned}
& 0=\left[\left[y x^{k-2-p^{\prime}}\right]\left[y x^{j+p^{\prime}+1} y\right]\right]=\left[\left[y x^{k-2-p^{\prime}}\right]\left[y x^{j+p^{\prime}+1}\right] y\right] \\
& =-\left(\begin{array}{c}
j+p^{t}+1 \\
p^{\imath}
\end{array}\right)\left[y x^{k-2} y x x^{j} y\right]+\left(\begin{array}{c}
j+p^{t}+1 \\
p^{t}+1
\end{array}\right)\left[y x^{k-2} x y x^{j} y\right] \\
& =-\left(\begin{array}{c}
j+p^{t}+2 \\
p^{t}+1
\end{array}\right)\left[v y x x^{j} y\right] \text {, }
\end{aligned}
$$

where

$$
-\left(\begin{array}{c}
j+p^{t}+2 \\
p^{t}+1
\end{array}\right)=-\left(\begin{array}{c}
\beta p^{t}+p^{t}-1 \\
p^{t}+1
\end{array}\right) \equiv \beta \not \equiv 0 \quad(\bmod p)
$$

Note that even if $p^{t}=1$, the resulting coefficient is

$$
-\left(\begin{array}{l}
\beta \\
2
\end{array}\right)=-\left(\begin{array}{c}
j+3 \\
2
\end{array}\right)
$$


so that this also covers the case when $j+1 \equiv 0(\bmod p)$.

Now if $n>1$ we have $\left[y x^{2 q-3} y\right]=0$, so that

$$
\begin{aligned}
0 & =\left[\left[y x^{k-1-q}\right]\left[y x^{2 q-3} y\right]\right]=\left[\left[y x^{k-1-q}\right]\left[y x^{2 q-3}\right] y\right] \\
& =\left(\begin{array}{c}
2 q-3 \\
q-1
\end{array}\right)\left[y x^{k-2} y x x^{q-3} y\right]-\left(\begin{array}{c}
2 q-3 \\
q
\end{array}\right)\left[y x^{k-2} x y x^{q-3} y\right] \\
& =\left(\begin{array}{c}
2 q-2 \\
q
\end{array}\right)\left[v y x x^{q-3} y\right],
\end{aligned}
$$

where

$$
\left(\begin{array}{c}
2 q-2 \\
q
\end{array}\right)=\left(\begin{array}{c}
q+q-2 \\
q
\end{array}\right) \equiv 1 \quad(\bmod p)
$$

Also, using $\left[y x^{2 q-2} y\right]=0$ we get

$$
\begin{aligned}
0 & =\left[\left[y x^{k-1-q}\right]\left[y x^{2 q-2} y\right]\right]=\left[\left[y x^{k-1-q}\right]\left[y x^{2 q-2}\right] y\right] \\
& =\left(\begin{array}{c}
2 q-2 \\
q-1
\end{array}\right)\left[y x^{k-2} y x x^{q-2} y\right]-\left(\begin{array}{c}
2 q-2 \\
q
\end{array}\right)\left[y x^{k-2} x y x^{q-2} y\right] \\
& =\left(\begin{array}{c}
2 q-1 \\
q
\end{array}\right)\left[v y x x^{q-2} y\right],
\end{aligned}
$$

where

$$
\left(\begin{array}{c}
2 q-1 \\
q
\end{array}\right)=\left(\begin{array}{c}
q+q-1 \\
q
\end{array}\right) \equiv 1 \quad(\bmod p)
$$

Finally, for $h=(k+q-2) / 2$ we have

$$
\begin{aligned}
0 & =\left[\left[y x^{h}\right]\left[y x^{h}\right]\right] \\
& = \pm\left(\left(\begin{array}{l}
h \\
q
\end{array}\right)\left[y x^{k-2} y x x^{q-2} x\right]-\left(\begin{array}{c}
h \\
q-1
\end{array}\right)\left[y x^{k-2} x y x^{q-2} x\right]\right) \\
& = \pm\left(\begin{array}{c}
h+1 \\
q
\end{array}\right)\left[y x^{k-2} y x x^{q-2} x\right],
\end{aligned}
$$

where

$$
\left(\begin{array}{c}
h+1 \\
q
\end{array}\right)=\left(\begin{array}{c}
q n+(q-3) / 2 \\
q
\end{array}\right) \equiv n \neq 0 \quad(\bmod p) .
$$

It follows that the component of $L$ of weight $k+q$ vanishes, so that $L$ is finite dimensional, as claimed. 


\section{The case when all diamonds are of type $\infty$}

In this section we consider (-1)-algebras $L$ in which all diamonds are of type $\infty$. This is defined as follows, and extends a definition we have given for the second diamond. As in Section 3, choose a non-zero element $y \in C_{L_{1}}\left(L_{2}\right)$, and an arbitrary element $x \in L_{1}$, independent of $y$. Suppose there is a diamond in $L$ in weight $h>1$. Choose a non-zero element $u$ of weight $h-1$. We say that this diamond is of type $\infty$ if

$$
[u x y]+[u y x]=0, \quad[u x x]=[u y y]=0 .
$$

As in Section 3, one sees that this definition is independent of the choice of $y \in C_{L_{1}}\left(L_{2}\right)$ and $x$.

These algebras turn out to have a strong connection to algebras of maximal class, as studied in $[8,10,15]$. We refer to [8] for all facts about algebras of maximal class mentioned without reference in the following.

Throughout this section $p$ will be an odd prime. We recall that an algebra of maximal class is a graded Lie algebra

$$
M=\bigoplus_{i=1}^{\infty} M_{i}
$$

over a field $\mathbf{F}$ of characteristic $p$, where $\operatorname{dim}\left(M_{1}\right)=2, \operatorname{dim}\left(M_{i}\right) \leq 1$, for $i \geq 2$, and $\left[M_{i} M_{1}\right]=M_{i+1}$, for all $i \geq 1$, or equivalently, $M_{1}$ generates $M$ as an algebra. All subalgebras and ideals will be taken to be graded. A two-step centralizer [8] in $M$ is a one-dimensional suspace of $M_{1}$ of the form $C_{M_{1}}\left(M_{i}\right)$, for some $i \geq 2$. We choose an element $y \in M_{1}$ such that $y$ spans the first two-step centralizer, that is, $\langle y\rangle=C_{M_{1}}\left(M_{2}\right)$.

Let $M$ be an infinite-dimensional algebra of maximal class. Consider a maximal graded subalgebra $L$ of $M$. Such an $L$ is of the form $M(z)=\langle z\rangle+M^{2}$, where $0 \neq z \in M_{1}$, and $M^{2}=[M M]=\bigoplus_{i \geq 2} M_{i}$. Clearly $L$ inherits a grading from $M$. We want to study which of these algebras $L$ are thin, with respect to a suitable new grading. In fact, all homogeneous components of $L$ have dimension 1 in the grading induced by $M$, so $L$ cannot be thin with respect to this grading.

To begin with, $M(z)$ has to be two-generated, so it has to be generated by $z$ and $[y x]$. We thus try and reassign weight 1 to both $z$ and $[y x]$, and investigate for which $z$ does this assignment makes $M(z)$ into a graded Lie algebra, and a thin one.

Note that $M(y)$ is not thin, as $[y x y]=0$. If $\langle y\rangle$ is the only two-step centralizer, then $M$ is metabelian, and all maximal subalgebras other than $M(y)$ are again of maximal class. This holds true more generally for all $M(z)$, when $z$ is not in any two-step centralizer, because then $\left[M_{i} z\right]=M_{i+1}$ for all $i \geq 2$. 
Suppose now $M$ has at least two distinct two-step centralizers, and let $\langle x\rangle$ be the second one in order of occurrence. We show that if $\langle z\rangle$ is a two-step centralizer other than $\langle y\rangle$ or $(x)$, then $M(z)$ is not thin.

Suppose it is. Without loss of generality, we may assume $z=x+y$. Then the thin grading has to be obtained by taking $z$ and $[y z]$ to be of weight 1 . We have then that [yzz] acquires weight 2 , and so on. Let now $M_{h}$ be the first homogeneous component centralized by $x$. Let $0 \neq u \in M_{h-1}$, so that $u$ has weight $h-2$ in $M(z)$, and

$$
[u y]=0, \quad[u z]=[u x] \neq 0, \quad[u x x]=0, \quad[u x y] \neq 0 .
$$

As $[u[y z]]=[u y z]-[u z y]=-[u x y]$, we see that the independent elements $[u x]$ and $[u x y]$ acquire the same weight $h-1$ in $M(z)$, so that we have a diamond in $M(z)$ in this weight. But $[[u x] z]=[u x y]$ acquires also weight $h \neq h-1$, a contradiction.

We have proved part of

THEOREM 2. Let $M$ be an infinite-dimensional algebra of maximal class, and let $\langle y\rangle=C_{M_{1}}\left(M_{2}\right)$ be its first two-step centralizer.

(1) The maximal subalgebra $M(y)$ is not thin.

(2) If $z \in M_{1}$ is not in any two-step centralizer, then $M(z)$ is of maximal class, if one takes $z$ and $[y z]$ to be of weight one.

(3) If $M$ has more than two distinct two-step centralizers, then no maximal subalgebra is thin.

(4) If $M$ has exactly two distinct two-step centralizers, and $\langle x\rangle$ is the second twostep centralizer in order of occurrence, then no subalgebra $M(z)$, with $\langle z\rangle \neq\langle x\rangle$, is thin. However, if $\langle z\rangle \neq\langle x\rangle,\langle y\rangle$, then $M(z)$ is of maximal class.

(5) If $M$ has exactly two distinct two-step centralizers, and $\langle x\rangle$ is the second twostep centralizer, then $M(x)$ is a (-1)-algebra with all diamonds of type $\infty$.

PROOF. Let $M$ be an algebra of maximal class with at least two distinct two-step centralizers, and let $\langle x\rangle$ be the second one. We complete the proof of the theorem by proving that $M(x)$ is a thin algebra, and it has the required type, if and only if $M$ has exactly two distinct two-step centralizers.

Let $M_{h}$ be the first homogeneous component of $M$ centralized by $x$, and let $0 \neq$ $u \in M_{h-1}$. Note that $0 \neq\left[y x^{h-2}\right]=\left[[y x] x^{h-3}\right] \in M_{h-1}$, so that $u$ has weight $h-2$ in the thin grading of $M(x)$. We have

$$
[u x] \neq 0 \quad \text { and } \quad[u[y x]]=-[u x y] \neq 0,
$$

so that $M(x)$ has a diamond in weight $h-1$. Also,

$$
[u x x]=0, \quad[u[y x][y x]]=[u x y x y]=0,
$$




$$
[u x[y x]]=[u x y x] \neq 0 \quad \text { and } \quad[u[y x] x]=-[u x y x],
$$

so that this is indeed a diamond of type $\infty$.

The two equalities on the second line follow from basic properties of the so-called constituents in graded Lie algebras of maximal class, as $p>2$. See [8] for details.

The same argument shows that each homogeneous component centralized by $x$ gives rise to a diamond in $M(x)$, and that homogeneous elements in $M$ stay homogeneous in $M(x)$. However, if $M$ has a two-step centralizer $\langle z\rangle$ other than $\langle y\rangle$ and $\langle x\rangle$, say $z=x+y$, then the grading is destroyed when we reach the first homogeneous component $M_{h}$ of $M$ centralized by $z$. This is basically the same argument we have seen above. Let $u \in M_{h-1}$, so that $[u z]=[u x] \neq 0$, and $[u x z]=0$, so that $[u x x]=-[u x y] \neq 0$. Then the latter element acquires two distinct weights from

$$
[u x x]=[u[y x]],
$$

a contradiction.

To prove the converse (Theorem 4 below), we first need a technical lemma.

LEMMA 3. Let $L$ be an infinite-dimensional thin algebra with all diamonds of type $\infty$, and second diamond in weight $2 q-1$, for some power $q$ of the characteristic $p$ of the underlying field. Choose $0 \neq y \in L_{1}$ such that $\left[L_{2} y\right]=0$, and any $x \in L_{1} \backslash\langle y\rangle$. Let $u$ be a non-zero homogeneous element of $L$, of weight $k$. Suppose $[u y]=0$, but $[u x y] \neq 0$. Then $[u x x]$ and $[u x y]$ are linearly independent, so that there is a diamond in weight $k+2$.

PROOF. We proceed by induction on $k$. From our assumption, our statement holds for $k \leq 2 q$. To provide the induction step, we may suppose there is a diamond in weight $k-1$. Let $v$ be a non-zero homogeneous element of weight $k-2$. By assumption we have $[v x y]=-[v y x]$, and $[v x x]=[v y y]=0$.

Suppose

$$
0=[v y y]=[v y x y]=\cdots=\left[v y x^{l} y\right],
$$

but $\left[v y x^{l+1} y\right] \neq 0$. Let $u=\left[v y x^{l}\right]$. If we show that $[u x x]$ and $[u x y]$ are linearly independent, we have the induction step.

Suppose this is not the case, and $[u x x]=\lambda[u x y]$ for some $\lambda$. Redefining $x$ to be $x-\lambda y$, we may assume $[u x x]=0$. We show that this leads to a contradiction.

Note that we have $\left[y x^{m} y\right]=0$ for all values of $m \neq 2 q-2,2 q-3$. We actually have already $\left[y x^{m}\right]=0$ for $m \geq 2 q-1$.

We first show that $l \geq q-3$. In fact, if $l<q-3$, we may compute

$$
0=\left[v\left[y x^{l+1} y\right]\right]=\left[v\left[y x^{l+1}\right] y\right]-\left[v y\left[y x^{l+1}\right]\right]=\left(1+(l+1)+(-1)^{l+1}\right)[u x y] .
$$


This gives $[u x y]=0$, a contradiction, unless $l$ is odd, and $l+3 \equiv 0(\bmod p)$, or $l$ is even, and $l+1 \equiv 0(\bmod p)$.

Write $l+3=\beta p^{t}$, with $\beta \neq 0(\bmod p)$. Note $p^{t}<q$, as $l<q-3$, so that $l+1+p^{t}<2 q-3$. In various places it is handy to write something like $v^{-k}$, with $k>0$ for a homogeneous element such that $\left[v^{-k} x^{k}\right]=v$. Suppose first $t>0$, and compute

$$
0=\left[v^{-p^{t}}\left[y x^{l+1+p^{t}} y\right]\right]=\left[v^{-p^{t}}\left[y x^{l+1+p^{t}}\right] y\right]= \pm\left(\begin{array}{c}
l+2+p^{t} \\
p^{t}+1
\end{array}\right)[u x y]=-\beta[u x y] .
$$

If $t=0$, that is, $l+3 \neq \equiv 0(\bmod p)$, then we may assume $l+1 \equiv 0(\bmod p)$, and thus the binomial coefficient above evaluates to

$$
\left(\begin{array}{c}
l+3 \\
2
\end{array}\right)=\frac{(l+3)(l+2)}{2} \equiv 1 \quad(\bmod p) .
$$

We also have $l \leq 2 q-2$. This follows from

$$
0=\left[v x\left[y x^{2 q-1}\right]\right]=\left[v x y x^{2 q-1}\right]=-\left[v y x^{2 q-1} x\right],
$$

so $l+1 \leq 2 q-1$.

We now obtain a contradiction, by proving $L$ to be infinite-dimensional. This follows from

$$
0=[u x y y]=[u x y x y]=[u x y x x y]=\cdots=\left[u x y x^{q / p} y\right],
$$

and

$$
\left[u x y x^{k}\right]=0, \quad \text { for some } k \leq q / p .
$$

We first have

$$
0=[u[x y y]]=[u x y y] .
$$

More generally, let $m=\beta p^{t}$, with $\beta \not \equiv 0(\bmod p)$. Suppose first $t>0$, and compute

$$
0=\left[u^{-p^{\prime}+1}\left[y x^{m+p^{\prime}-1} y\right]\right]=-\beta\left[u x y x^{m-1} y\right] .
$$

This works for all values of $m$, as $m+p^{t}-1 \equiv-1(\bmod p)$. If $t=0$ the coefficient is $m \neq 0(\bmod p)$. This holds now for $m<2 q-3$, that is, for $m-1 \leq 2 q-5$. Note $2 q-5 \geq q / p$ for $p$ odd, so that (4) holds.

To prove (5), we distinguish some cases, according to the value of $l$.

If $l=q-3$, we compute

$$
0=\left[v\left[y x^{q-1} y\right]\right]=\left[v\left[y x^{q-1}\right] y\right]-\left[v y\left[y x^{q-1}\right]\right]
$$




$$
=\left[v y x^{q-1} y\right]+\left[v x y x^{q-2} y\right]-\left[v y x^{q-2} y x\right]-\left[v y x^{q-1} y\right]=-[u x y x] .
$$

This is (5) for $k=1$.

Now let $l$ be arbitrary. If $l \neq 2 q-4,2 q-5$, we have $\left[y x^{l+2} y\right]=0$, so that we can compute

$$
0=\left[v\left[y x^{l+2} y\right]\right]= \pm(l+2)\left[v y x^{l+1} y x\right]= \pm(l+2)[u x y x] .
$$

This is $(5)$ for $k=1$, unless $l+2 \equiv 0(\bmod p)$. In this case, write $l+2=\beta p^{t}$, with $\beta \not \equiv 0(\bmod p)$, and $t>0$. Note that $p^{t}<q$, and $l+1+p^{t} \equiv-1(\bmod p)$, so that $\left[y x^{l+1+p^{l}} y\right]=0$. We compute

$$
0=\left[v\left[y x^{l+1+p^{\prime}} y\right]\right]= \pm\left(\begin{array}{c}
\beta p^{\prime}+p^{\prime}-1 \\
p^{\prime}
\end{array}\right)\left[v y x^{l+1} y x^{p^{\prime}}\right]= \pm \beta\left[u x y x^{p^{\prime}}\right] .
$$

This is (5) for $k=p^{t} \leq q / p$.

We now deal with the two remaining cases. If $l=2 q-4$ it is enough to compute

$$
0=\left[v\left[y x^{l+3} y\right]\right]= \pm\left(\begin{array}{c}
l+3 \\
2
\end{array}\right)\left[v y x^{l+1} y x x\right]= \pm[u x y x x],
$$

which is (5) for $k=2$. If $l=2 q-5$ we compute

$$
0=\left[v\left[y x^{l+4} y\right]\right]= \pm\left(\begin{array}{c}
l+4 \\
3
\end{array}\right)\left[v y x^{l+1} y x x x\right]= \pm[\operatorname{uxyxxx}],
$$

which is (5) for $k=3$.

THEOREM 4. Let $L$ be an infinite-dimensional thin algebra with all diamonds of type $\infty$. Then there is a grading of $L$, different from the original one, and a derivation $D$ of $L$, which is of weight 1 with respect to this new grading, such that the extension $M=L+(D)$ is an algebra of maximal class. In particular, $L$ is a (-1)-algebra.

PROOF. We choose a homogeneous basis $\mathscr{B}$ of $L$ in the following way. We choose $0 \neq y \in L_{1}$ with the property that $\left[L_{2} y\right]=0$, and any $x \in L_{1} \backslash\langle y\rangle$, and take $x$ and $y$ to be part of the basis. Proceeding by induction on the weight, if the homogeneous element $u$ of weight $k$ is in the basis, and there is no diamond in weight $k+1$, then we add $[u x]$ to the basis, according to Lemma 3 , and continue with $u$ replaced with $[u x]$. If there is a diamond in weight $k+1$, then we add the elements $[u x],[u y],[u y x]$ to the basis, and restart the procedure replacing $u$ with [uyx].

Now we define a linear map $D$ on $L$ by specifying it on $\mathscr{B}$. We show that $D$ is a derivation, and that it has weight 1 with respect to a suitable new grading of $L$. This proves our claim. 
We define $D$ in the following way

$$
\begin{cases}D x=y, & \\ D u=[v y] & \text { if } u=[v x] \text { for some } v \in \mathscr{B} \text { such that } 0 \neq[v y] \in \mathscr{B} \\ D u=0 & \text { otherwise. }\end{cases}
$$

The second possibility arises when $v$ lies just before the diamond.

To prove that $D$ is a derivation, we have to check that for all $u, v \in \mathscr{B}$

$$
D[u w]=[D u, w]+[u, D w]
$$

holds. Proceeding by induction on the minimum of the weights of $u$ and $w$, we see that it is enough to show this when $w \in\langle x, y\rangle$.

As $D$ is nearly always zero, there are only a few cases to be checked.

(1) $w=x$, and $u=v$ is just before a diamond. We have $D w=D x=y$, and $D[u w]=D[v x]=[v y]$, and in fact $[D u, w]+[u, D w]=[D v, x]+[v y]=[v y]$.

(2) $w=x$, and $u=[v x]$ for some $v$ just before a diamond. Here we have $[u w]=$ $[v x x]=0$, and $[D u, w]+[u, D w]=[D[v x], x]+[v x y]=[v y x]+[v x y]=0$.

(3) $w=y$, and $u=[v x]$ for some $v$ just before a diamond. Here $D[u w]=$ $D[v x y]=0$, and $[D u, w]+[u, D w]=[v y y]=0$.

Therefore, it is clear that we have exploited the whole definition of a diamond of type $\infty$ here.

We now grade the extension $M=L+\langle D\rangle$ by assigning weight 1 to $x$ and $D$. As $D$ is nonzero precisely on those elements of the basis $\mathscr{B}$ on which ad $(x)$ vanishes, we see that $x$ and $D$ span $M$, that this assigns unique weights to the elements of $\mathscr{B}$, and that each homogeneous component of weight greater than 1 is one-dimensional, so that $M$ is of maximal class.

Note that with respect to this new grading $x$ takes weight 1 , and $y$ takes weight 2, so this induces a grading on $L$ that is different from the original one.

Note that over any field $\mathbf{F}$ of positive characteristic there are $2^{\aleph_{0}}$ algebras of maximal class with two distinct two-step centralizers, and that these algebras can be classified (see $[10,15]$ for details). Also, among these algebras we have (periodic) soluble ones, periodic insoluble, and (insoluble) non-periodic ones. Therefore, we have a similar variety among the $(-1)$-algebra with all diamonds of type $\infty$.

\section{Finite diamonds}

We now consider (-1)-algebras in which all diamonds are of finite type, as defined in the following. Let $T$ be a $(-1)$-algebra over the field $\mathbf{F}$, where the second diamond 
occurs in weight $k=-1+2 q$, where $q$ is a power of the characteristic $p$ of the field $\mathbf{F}$, and suppose the second diamond is of finite type.

We always work over a field of characteristic $p>2$; later we assume that $p>3$.

We have, in the notation of the preliminaries,

$$
[v y x]=\lambda[v x x],
$$

where $0 \neq y \in T_{1}$ is defined by $\left[T_{2} y\right]=0$, the element $x \in L_{1}$ is independent of $y$, but otherwise arbitrary, and $v=\left[y x^{2 q-3}\right]$. By renaming $y$ to be $\lambda^{-1} y$, we see that we can rewrite the above as

$$
[v y x]=[v x x]
$$

We see that the thin Lie algebra $T$ is now uniquely determined. To describe $T$, we need to fix some conventions.

From now on we keep $y$ and $x$ fixed. Suppose in $T$ there is a diamond in some weight $h>1$, and let $u$ be a homogeneous element of weight $h-1$. Suppose

$$
[u x y]+[u y x]=0, \quad[u y y]=0, \quad[u y x]=\lambda[u x x],
$$

for some $\lambda$. Then we say that this diamond has finite type $\lambda$. Thus in the above we have arranged the second diamond to be of type 1 .

Now $T$ have diamonds in all weights congruent to $1(\bmod q-1)$, the diamond in weight $1+\lambda(q-1)$ being of type $\lambda-1$. Thus the types run over the elements of the base field $\mathbf{F}_{p}$, so that in particular $T$ is defined over $\mathbf{F}_{p}$, and it is periodic. We note that if $\lambda \equiv 1(\bmod p)$, and $v$ is a nonzero element of weight $\lambda(q-1)$, then

$$
[v y x]=(\lambda-1)[v x x]=0,
$$

so that in this case we do not have a real diamond, but a degenerate one, as the element $[v y]$ is central.

Our main result can be stated as follows.

THEOREM 5. Let $\mathbf{F}$ be a field of characteristic $p>5$, and let $q$ be a power of $p$. Then there is uniquely determined, infinite-dimensional (-1)-algebra $T$ with second diamond in weight $2 q-1$, and second diamond of finite type. In $T$ all diamonds are of finite type. One can prescribe the second diamond to have type 1. Then the types of the diamonds run cyclically through the integers $0,1, \ldots, p-1$ modulo $p$.

To establish uniqueness of the algebra $T$, we proceed as in [5]. We construct first a particular finite-dimensional simple algebra $A$, according to the methods of Albert and Frank [1]. We exhibit certain cocycles of $A$, which are independent in the second 
cohomology group $H^{2}(A, \mathbf{F})$, and consider the corresponding extension $\widehat{A}$. We then consider a certain loop algebra $N$ of $\widehat{A}$. The algebra $N$ has an infinite-dimensional centre $Z(N)$, inherited by that of $\widehat{A}$. We show that $N / Z(N)$ is thin, with second diamond of type 1 in weight $-1+2 q$. We also show that $N$ is finitely presented. More precisely, prescribing the structure of $N$ up to weight $2 q$ is enough to determine $N$ completely. It follows that $T=N / Z(N)$ is the unique infinite-dimensional thin algebra with second diamond of type 1 in weight $-1+2 q$.

As in [5], we may note that $T=N$ itself is not finitely presented, by the following result of B. H. Neumann ([23], see also [24, 2.2.3]).

THEOREM 6. Let $L$ be a finitely presented Lie algebra, and $M$ be a finitely generated Lie algebra $M$. Suppose there is a surjective morphism

$$
g: M \rightarrow L
$$

Then $\operatorname{ker}(g)$ is finitely generated as an ideal of $M$.

In fact, taking $M=N, L=T=N / Z(N)$, and $\varphi$ to be the natural epimorphism, we see that $\operatorname{ker}(g)=Z(N)$ is not finitely generated as an ideal, thus preventing $T$ from being finitely presented. By the same argument, the fact that $N$ is finitely presented implies that our cocycles are a basis of $H^{2}(A, \mathbf{F})$.

\section{Constructing the algebra with all diamonds of finite type}

Let $p$ be an odd prime, $q$ a power of $p$. Consider the $\mathbf{F}_{p}$-vector space $S=\mathbf{F}_{p} \oplus \mathbf{F}_{q}$, and the $\mathbf{F}_{p}$-bilinear, alternating map

$$
\begin{aligned}
& \vartheta: S \times S \rightarrow \mathbf{F}_{q} \\
& ((u, \alpha),(v, \beta)) \mapsto v \alpha-u \beta .
\end{aligned}
$$

Consider the $\mathbf{F}_{q}$-vector space $A$ with basis $f_{s}, s \in S^{*}$. Now $A$ becomes a Lie algebra over $\mathbf{F}_{q}$ defining

$$
\left[f_{s} f_{t}\right]=\vartheta(s, t) f_{s+t}
$$

where we understand $f_{0}=0$. This is an instance of the algebras constructed by Albert and Frank in [1]. It would not be difficult to see, with the methods of [1], that $A$ is a simple Lie algebra. We prove this, however, in the related paper [7], by identifying it with a non-graded Hamiltonian algebra, as mentioned in the introduction. 
In the following we simply write $f_{u, \alpha}$ for $f_{(u, \alpha)}$. Easy direct calculations show that the following $n+1$ maps are cocycles for the algebra $A$ :

$$
\varphi_{r}\left(f_{u, \alpha}, f_{v, \beta}\right)= \begin{cases}\alpha^{p^{r}} & \text { if } \alpha+\beta=0 \text { and } u+v=0 \\ 0 & \text { otherwise }\end{cases}
$$

for $1 \leq r \leq n$, where $q=p^{n}$, and

$$
\psi\left(f_{u, \alpha}, f_{v, \beta}\right)= \begin{cases}u & \text { if } u+v=0 \text { in } \mathbf{F}_{p}, \\ 0 & \text { otherwise. }\end{cases}
$$

To prove that these cocycles are independent in the cohomology group, consider the vector space

$$
\widehat{A}=A \oplus \bigoplus_{r=1}^{n}\left\langle z_{r}\right\rangle \oplus\langle w\rangle
$$

turn it into a Lie algebra by declaring the $z_{r}$ and $w$ to be central, and defining a Lie bracket via

$$
\llbracket f_{s} f_{t} \rrbracket=\left[f_{s} f_{t}\right]+\sum_{r=1}^{n} \varphi_{r}\left(f_{s}, f_{t}\right) z_{r}+\psi\left(f_{s}, f_{t}\right) w .
$$

(See [14] or [11] for the easy cohomological background.) In the next section we show that $w$ and the $z_{r}$ lie in the derived subalgebra of $\widehat{A}$.

\section{A thin grading}

From now on we assume $p>3$. Consider the $\mathbf{F}_{p}$-subalgebra $B$ of $\widehat{A}$ generated by

$$
x=f_{1,0}, \quad y=\sum_{\alpha \in \mathbf{F}_{q}} f_{1, \alpha} .
$$

We revert to the usual Lie bracket $[\cdot, \cdot]$ here. We have first

$$
[y x]=\sum_{\alpha \in \mathbf{F}_{q}}\left[f_{1, \alpha}, f_{1,0}\right]=\sum_{\alpha \in \mathbf{F}_{q}} \alpha f_{2, \alpha},
$$

and in general, proceeding by induction

$$
\left[y x^{j-1}\right]=\sum_{\alpha \in \mathbf{F}_{q}} \alpha^{j-1} f_{j, \alpha}-\sum_{\alpha \in \mathbf{F}_{q}} \alpha^{j-2} \delta_{p}(j, 0) w .
$$


Note that

$$
\left[y x^{(q-1) p}\right]=\sum_{\alpha \in \mathbf{F}_{q}} \alpha^{(q-1) p} f_{1+(q-1) p, \alpha}=\sum_{\alpha \in \mathbf{F}_{q}^{*}} f_{1, \alpha}=y-x
$$

so that the elements $\left[y x^{j-1}\right]$ are periodic in $j$, of period $(q-1) p$.

Note that $w$ occurs with a nonzero coefficient if and only if $j \equiv 0(\bmod p)$, and $j-2 \equiv 0(\bmod q-1)$. This holds in the range $1, \ldots,(q-1) p$ when $j=2 q$. Here we get

$$
\left[y x^{2 q-1}\right]=\sum_{\alpha \in \mathbf{F}_{q}} \alpha f_{0, \alpha}+w .
$$

We claim that all the elements we have obtained are independent over $\mathbf{F}_{q}$. Note that $w$ does not play a serious role here, as it occurs only once. Now the elements that have the same support $\left\{f_{j, \alpha}: \alpha \in \mathbf{F}_{q}\right\}$, for a given $j$, are the

$$
\left[y x^{j-1+p i}\right]=\sum_{\alpha \in \mathbf{F}_{q}} \alpha^{j-1+p i} f_{j, \alpha}, \quad \text { for } 0 \leq i<q-1 .
$$

These elements have coefficient matrix

$$
\left[\alpha^{j-1+p i}\right]_{(\alpha, i)}, \quad \text { for } \alpha \in \mathbf{F}_{q}^{*} \text {, and } 0 \leq i<q-1
$$

and we have

$$
\operatorname{det}\left(\left[\alpha^{j-1+p i}\right]_{(\alpha, i)}\right)=\left(\prod_{\alpha \in F_{q}^{*}} \alpha\right)^{j-1} \operatorname{det}\left(\left[\alpha^{p i}\right]_{(\alpha, i)}\right)=(-1)^{j-1} \operatorname{det}\left(\left[\left(\alpha^{p}\right)^{i}\right]_{(\alpha, i)}\right),
$$

a nonzero Vandermonde determinant.

Now we compute

$$
\begin{aligned}
{\left[\left[y x^{j-1}\right] y\right]=} & {\left[\sum_{\alpha \in \mathbf{F}_{q}} \alpha^{j-1} f_{j, \alpha}, \sum_{\beta \in \mathbf{F}_{q}} f_{1, \beta}\right] } \\
= & \sum_{\alpha, \beta \in \mathbf{F}_{q}} \alpha^{j-1}(-j \beta+\alpha) f_{j+1, \alpha+\beta}-\sum_{\alpha, \beta \in \mathbf{F}_{q}} \alpha^{j-1} \delta_{p}(j,-1) w \\
& +\sum_{r=0}^{n-1} \delta_{p}(j,-1)\left(\sum_{\alpha \in \mathbf{F}_{q}} \alpha^{j-1+p^{r}}\right) z_{r} .
\end{aligned}
$$

Note first that

$$
\sum_{\alpha, \beta \in \mathbf{F}_{q}} \alpha^{j-1}(-j \beta+\alpha) f_{j+1, \alpha+\beta}=\sum_{\alpha, \gamma \in \mathbf{F}_{q}} \alpha^{j-1}(-j \gamma+(j+1) \alpha) f_{j+1, \gamma}
$$




$$
=-j \sum_{\gamma \in \mathbf{F}_{q}} \gamma\left(\sum_{\alpha \in \mathbf{F}_{q}} \alpha^{j-1}\right) f_{j+1, \gamma}+(j+1) \sum_{\gamma \in \mathbb{F}_{q}}\left(\sum_{\alpha \in \mathbf{F}_{q}} \alpha^{j}\right) f_{j+1, \gamma} .
$$

Also note that the coefficient of $w$ is always zero, as $\sum_{\beta \in \mathbf{F}_{q}} 1=0$.

The sums $\sum_{\alpha \in \mathbf{F}_{q}} \alpha^{j-1}$ and $\sum_{\alpha \in \mathbf{F}_{q}} \alpha^{j}$ vanish, except in the following cases, which give rise to diamonds. When $j=\lambda(q-1)$ for some $\lambda$, then $\sum_{\alpha \in \mathbf{F}_{q}} \alpha^{j}=-1$, and

$$
\left[y x^{\lambda(q-1)-1}\right]=\sum_{\alpha \in \mathbf{F}_{q}^{*}} \alpha^{-1} f_{j, \alpha}
$$

is an element just before a diamond. We have here

$$
\left[y x^{\lambda(q-1)-1} y\right]=(\lambda-1) \sum_{\gamma \in \mathbf{F}_{q}} f_{-\lambda+1, \gamma}+\sum_{r=1}^{n} \delta_{p}(\lambda, 1)\left(\sum_{\alpha \in \mathbf{F}_{q}} \alpha^{p^{r}-1}\right) z_{r}
$$

The second term always vanishes, except when $\lambda=1$. In this case only the summand for $r=n$ survives, and the first term vanishes to give

$$
\left[y x^{q-2} y\right]=-z_{n}
$$

This shows that $z_{n}$ lies in the derived subalgebra of $\widehat{A}$; we do not have a real diamond here, as this element is of course central. For $\lambda \neq 1$ we have

$$
\left[y x^{\lambda(q-1)-1} y x\right]=(\lambda-1) \sum_{\gamma \in \mathbf{F}_{q}} \gamma f_{-\lambda+2, \gamma}-(\lambda-1) \sum_{\gamma \in \mathbb{F}_{q}} \delta_{p}(\lambda, 2) w,
$$

where the coefficient of $w$ is again always zero.

When $j-1=\lambda(q-1)$ for some $\lambda$, then $\sum_{\alpha \in \mathbf{F}_{q}} \alpha^{j-1}=-1$, and

$$
\left[y x^{\lambda(q-1)}\right]=\sum_{\gamma \in \mathbb{F}_{q}^{*}} f_{-\lambda+1, \gamma}
$$

Also

$$
\left[y x^{\lambda(q-1)} y\right]=(1-\lambda) \sum_{\gamma \in \mathbb{F}_{q}} \gamma f_{-\lambda+2, \gamma}=-\left[y x^{\lambda(q-1)-1} y x\right]=(1-\lambda)\left[y x^{\lambda(q-1)+1}\right],
$$

so this is a diamond of type $\lambda-1$, except when $\lambda=2$. Here we get, as we have seen,

$$
\left[y x^{2 q-1}\right]=\sum_{\alpha \in \mathbf{F}_{q}} \alpha f_{0, \alpha}+w=-\left[y x^{2 q-2} y\right]+w .
$$

This also shows that $w$ lies in the derived subalgebra of $\widehat{A}$. 
Finally, the coefficient

$$
\delta_{p}(j,-1) \sum_{\alpha \in \mathbf{F}_{q}} \alpha^{j-1+p^{r}}
$$

of $z_{r}$ always vanishes, unless $j \equiv-1(\bmod p)$ and $q-1$ divides $j-1+p^{r}$. We have then

$$
j=2(q-1)-p^{r}+1
$$

and the coefficient is -1 . We obtain

$$
\left[y x^{2 q-p^{r}-2} y\right]=-z_{r}
$$

for all $1 \leq r \leq n$ (this includes the case $r=n$, which we have already seen), and all the $z_{r}$ lie in the derived subalgebra.

We have obtained that the algebra $B$, which is defined over the prime field, has dimension

$$
\operatorname{dim}(B)=p(q-1)+p+n=p q+n=\operatorname{dim}(A)+n+1=\operatorname{dim}(\widehat{A})
$$

over $\mathbf{F}_{q}$. It follows that $\widehat{A}=B \otimes \mathbf{F}_{q}$, so that $\widehat{A}$ itself is defined over the prime field.

\section{The presentation}

The algebra $\widehat{A}$ is completely described by the following relations, that describe $\operatorname{ad}(x)$ and $\operatorname{ad}(y)$ :

$$
\begin{aligned}
& \begin{cases}{\left[v_{h-1} x x x^{j} y\right]=0} & \text { for } 0 \leq j<q-3, \text { if } h \neq \equiv 1 \quad(\bmod p), \\
{\left[v_{0} x^{q-p^{r}} y x\right]=0} & \text { for } 1 \leq r \leq n, \\
{\left[v_{0} x^{i} y\right]=0} & \text { for } 0<i<q-1, \text { and } i \neq q-p^{r},\end{cases} \\
& v_{h}=\left[v_{h-1} x^{q-1}\right], \\
& {\left[v_{h} y x\right]=-\left[v_{h} x y\right]}
\end{aligned}
$$

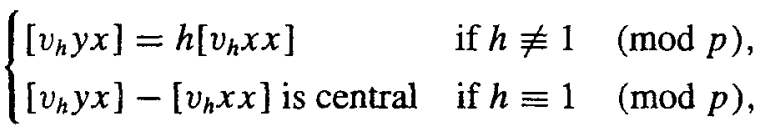$$
\left[v_{h} y y\right]=0 \text {. }
$$

Here the unlabeled relation $v_{h}=\left[v_{h-1} x^{q-1}\right]$ is just a recursive definition. The indices $h$ of the $v_{h}$ range over the non-negative integers regarded modulo $p$, where $p$ is a prime bigger than 3 . 
It is useful to define formally an element $v_{-1}$ such that $\left[v_{-1} x\right]=y-x,\left[v_{-1} y\right]=-y$; this provides some uniformity in the definitions. Modulo its centre

$$
\left\langle\left[v_{1} y x\right]-\left[v_{1} x x\right],\left[v_{0} x^{q-p^{r}} y x\right]: 1 \leq r \leq n\right\rangle,
$$

the algebra $\widehat{A}$ is graded over a cyclic group of order $(q-1) p$, and as such is thin, with diamonds

$$
\left\langle\left[v_{i} x\right],\left[v_{i} y\right]\right\rangle
$$

at weights congruent to $1(\bmod q-1)$. There is not really a diamond for $i \equiv 0$ $(\bmod p)$, as the element $\left[v_{0} y\right]$ is central. In $\widehat{A}$ there is another exceptional diamond after the one for $i \equiv 1(\bmod p)$, because here the elements $\left[v_{1} x x\right]$ and $\left[v_{1} y x\right]$ are not equal, but their difference is central.

Now we apply a twisted loop algebra procedure, that is, we take a tensor product

$$
\widehat{A} \otimes \mathbf{F}_{p}[t],
$$

where $\mathbf{F}_{p}[t]$ is a polynomial ring, and consider the subalgebra $N$ generated by $x \otimes t$ and $y \otimes t$. Abusing notation, we keep writing $x$ and $y$ for these two elements, and obtain an algebra with the same relations (6a)-(6d), the index $h$ now running over the integers, starting from -1 , and the element $v_{-1}$ is again used formally for uniformity in the definitions, as explained below.

With a further notational abuse, we take the algebra $L$ presented as

$$
\begin{aligned}
L=\langle x, y: & {\left[y x^{j} y\right]=0 \text { for } 0 \leq j<2 q-3, \text { and } } \\
& j \neq 2 q-p^{r}-2, \quad \text { for } 1 \leq r \leq n, \\
& {\left[y x^{2 q-p^{r}-2} y x\right]=\left[y x^{2 q-p^{-2}} x y\right]=0, \text { for } 1 \leq r \leq n, } \\
& {\left[y x^{2 q-3} y x\right]=-\left[y x^{2 q-3} x y\right] } \\
& {\left[\left[y x^{2 q-3} y x\right]-\left[y x^{2 q-3} x x\right], x\right]=0 } \\
& {\left.\left[y x^{2 q-1} y\right]\right\rangle . }
\end{aligned}
$$

Clearly $N$ is a homomorphic image of $L$, taking $x \mapsto x$, and $y \mapsto y$. We show that (7) implies all of (6a)-(6d). Here we take formally an element $v_{-1}$ with the property $\left[v_{-1} x\right]=y-x,\left[v_{-1} y\right]=-y$, and define the $v_{i}$ recursively via $v_{h}=$ $\left[v_{h-1} x^{q-1}\right]$.

Clearly (7) gives (6a)-(6d) for $h=0$, and for $h=1$ up to (6c). The relation (6d) then follows from

$$
0=\left[y x^{2 q-4}[y x y]\right]=-\left[y x^{2 q-3} y y\right] .
$$

Thus we proved (6a)-(6d) for values of $h$ up to some $i \geq 1$. Our task is to prove (6a)(6d) for $h=i+1$. 


\section{The hard case}

We deal first with the more difficult case $h \equiv 1(\bmod p)$, so let $i=h-1=t p$ for some $t$. As $i>0$, we have $i \geq p$. For (6a), we first compute, for $j<q-3$,

$$
\begin{aligned}
0 & =\left[v_{t p-1}\left[y x^{j+q} y\right]\right]=\left[v_{t p-1}\left[y x^{j+q}\right] y\right]-\left[v_{t p-1} y\left[y x^{j+q}\right]\right] \\
& =\left[v_{t p-1} y x^{j+q} y\right]-j\left[v_{t p-1} x y x^{j+q-1} y\right]-(-1)^{j+1}\left[v_{t p-1} y x^{j+q} y\right] \\
& =\left(1+j+(-1)^{j}\right)\left[v_{t p} x x x^{j} y\right],
\end{aligned}
$$

which fails to show what we need only for $j$ even, with $j+2 \equiv 0(\bmod p)$, and for $j$ odd, with $j \equiv 0(\bmod p)$.

Now take the element $u=\left[v_{t p-2} x^{q-2}\right]$ so that $[u x]=v_{t p-1}$. As in Section 5, in the following it is handy to use the notation $v^{-k}$, where $v$ is a homogeneous element, for a homogeneous element such that $\left[v^{-k} x^{k}\right]=v$. In this case, thus, we would write $u=v_{t p-1}^{-1}$.

We have

$$
\begin{aligned}
0 & =\left[u\left[y x^{j+q+1} y\right]\right]=\left[u\left[y x^{j+q+1}\right] y\right] \\
& =-(j+1)\left[v_{t p-1} y x x^{j+q-1} y\right]+\left(\begin{array}{c}
j+1 \\
2
\end{array}\right)\left[v_{t p-1} x y x^{j+q-1} y\right] \\
& =\frac{(j+1)(j+2)}{2}\left[v_{t p-1} x y x^{j+q-1} y\right],
\end{aligned}
$$

which again fails to show what we need only for $j+2 \equiv 0(\bmod p)$. In fact this is the place where all cocycles except one sneak in. Let $j+2=\beta p^{r}$, with $\beta \not \equiv 0$ $(\bmod p)$. We want to use the calculation

$$
\begin{aligned}
0= & {\left[v_{t p-1}^{-k}\left[y x^{j+q+p^{r}} y\right]\right] } \\
= & (-1)^{p^{r}}\left(\begin{array}{c}
j+q+p^{r} \\
p^{r}
\end{array}\right)\left[v_{t p-1} y x x^{j+q-1} y\right] \\
& +(-1)^{p^{r}+1}\left(\begin{array}{c}
j+q+p^{r} \\
p^{r}+1
\end{array}\right)\left[v_{t p-1} x y x^{j+q-1} y\right] \\
= & \pm\left(\begin{array}{c}
j+q+p^{r}+1 \\
p^{r}+1
\end{array}\right)\left[v_{t p-1} x x x^{j+q-1} y\right] \\
= & \pm \beta\left[v_{t p} x x x^{j} y\right] .
\end{aligned}
$$

This works unless

$$
j+q+p^{r}=q+(\beta+1) p^{r}-2=2 q-2,
$$


that is, unless $\beta=p^{n-r}-1$, or $j=q-p^{r}-2$. Here $r$ ranges from 1 to $n-1$. Note that for $r=n$ the corresponding statements

$$
\left[v_{t p} y x\right]=\left[v_{t p} x y\right]=0
$$

also hold, because of $(6 b)$ and $(6 c)$ in the previous iteration. Here we understand formally $\left[v_{t p} x \times x^{-2}\right]=v_{t p}$.

So we have to prove by backward induction on $r$

$$
\left[v_{t p} x^{q-p^{r}} y x\right]=\left[v_{t p} x^{q-p^{\prime}} x y\right]=0 .
$$

We start with

$$
\begin{aligned}
0= & {\left[v_{t p-1}\left[y x^{2 q-p^{r}-1} y\right]\right]=\left[v_{t p-1}\left[y x^{2 q-p^{r}-1}\right] y\right]-\left[v_{t p-1} y\left[y x^{2 q-p^{r}-1}\right]\right] } \\
= & {\left[v_{t p-1} y x^{2 q-p^{r}-1} y\right]+\left[v_{t p-1} x y x^{2 q-p^{r}-2} y\right] } \\
& -(-1)(-1)\left[v_{t p-1} y x^{2 q-p^{r}-2} y x\right]-\left[v_{t p-1} y x^{2 q-p^{r}-1} y\right] \\
= & {\left[v_{t p} x^{q-p^{r}} y x\right]+\left[v_{t p} x^{q-p^{r}} x y\right] . }
\end{aligned}
$$

The second calculation is

$$
\begin{aligned}
0 & =\left[v_{t p-2} x\left[y x^{3 q-p^{r}-3} y\right]\right] \\
& =\left[v_{t p-2} x\left[y x^{3 q-p^{r}-3} y\right]-\left[v_{t p-2} x y\left[y x^{3 q-p^{r}-3}\right]\right]\right. \\
& =\left[v_{t p-2} x y x^{3 q-p^{r}-3} y\right]-(-1)^{2}(-3)\left[v_{t p-2} x y \cdots y x\right]-(-1)^{1}\left[v_{t p-2} x y \cdots x y\right] .
\end{aligned}
$$

We have omitted terms whose coefficients

$$
\left(\begin{array}{c}
3 q-p^{r}-3 \\
q-1
\end{array}\right), \quad\left(\begin{array}{c}
3 q-p^{r}-3 \\
q-2
\end{array}\right), \quad\left(\begin{array}{c}
3 q-p^{r}-3 \\
q-3
\end{array}\right),
$$

vanish, as $p^{r}<q$. For instance

$$
\left(\begin{array}{c}
3 q-p^{r}-3 \\
q-3
\end{array}\right)=\left(\begin{array}{c}
2 q \\
0
\end{array}\right)\left(\begin{array}{c}
q-p^{r}-3 \\
q-3
\end{array}\right)=0
$$

as $p^{r}<q$. The total result is

$$
0=4\left[v_{t p} x^{q-p^{r}} x y\right]+6\left[v_{t p} x^{q-p^{r}} y x\right],
$$

which completes the proof of (6a).

The relation (6d) immediately follows from

$$
0=\left[\left[v_{t p} x^{q-2} x\right][y x y]\right]=-\left[v_{t p+1} y y\right] .
$$


We consider now (6b), that is,

$$
\left[v_{t p+1} x y\right]+\left[v_{t p+1} y x\right]=0 .
$$

This identity has weight $(t p+1+1)(q-1)+2=t p(q-1)+2 q$. Take

$$
\lambda=\frac{t p(q-1)+2 q}{2}-1=\frac{t p(q-1)}{2}+q-1 .
$$

Note that $\lambda \equiv-1(\bmod p)$. We want to expand $0=\left[\left[y x^{\lambda}\right]\left[y x^{\lambda}\right]\right]$. Consider first the case $t$ even, so that

$$
\lambda=(q-1)\left(\frac{t}{2} p+1\right)
$$

Note that in this case $\lambda$ is even, and $\left[y x^{\lambda}\right]=\left[v_{t p / 2} x\right]$ lies in a diamond of type zero.

We expand

$$
\begin{aligned}
0= & {\left[\left[y x^{\lambda}\right]\left[y x^{\lambda}\right]\right] } \\
= & \sum_{s=1}^{t p / 2}\left(-\left(\begin{array}{c}
\lambda \\
s(q-1)-1
\end{array}\right)\left[v_{t p / 2+s} y x x^{\lambda-s(q-1)-2}\right]\right. \\
& \left.+\left(\begin{array}{c}
\lambda \\
s(q-1)
\end{array}\right)\left[v_{t p / 2+s} x y x^{\lambda-s(q-1)-2}\right]\right)-\lambda\left[v_{t p+1} y x\right]+\left[v_{t p+1} x y\right] \\
= & -2 \sum_{s=1}^{t p / 2}\left(\frac{t}{2} p+s\right)\left(\begin{array}{c}
\lambda+1 \\
s(q-1)
\end{array}\right)\left[v_{t p+1} x x\right]+\left[v_{t p+1} y x\right]+\left[v_{t p+1} x y\right] .
\end{aligned}
$$

We have $\lambda+1 \equiv 0(\bmod p)$, so the first $p$-adic digit of $\lambda+1$ is zero. Now the first $p$-adic digit of $s(q-1)$ is the remainder of $-s(\bmod p)$. By Lucas' theorem, the binomial coefficient can only be nonzero when $s \equiv 0(\bmod p)$. But in this case it is the coefficient $t / 2 p+s$ that vanishes, so that we are left with (6b).

Now consider the case $t$ odd. This time we have

$$
\lambda=\frac{t p+1}{2}(q-1)+\frac{q-1}{2}
$$

and $\left[y x^{\lambda}\right]=\left[v_{(p+1) / 2} x^{(q+1) / 2}\right]$. We expand

$$
\begin{aligned}
0= & {\left[\left[y x^{\lambda}\right]\left[y x^{\lambda}\right]\right] } \\
= & \pm \sum_{s=1}^{(t p-1) / 2}\left(\left(\begin{array}{c}
\lambda \\
(q-1) / 2+s(q-1)-1
\end{array}\right)\left[v_{(t p+1) / 2+s} y x x^{\lambda-\cdots}\right]\right. \\
& \left.-\left(\begin{array}{c}
\lambda \\
(q-1) / 2+s(q-1)
\end{array}\right)\left[v_{(t p+1) / 2+s} x y x^{\lambda-\cdots}\right]\right)-\lambda\left[v_{t p+1} y x\right]+\left[v_{t p+1} x y\right]
\end{aligned}
$$




$$
\begin{aligned}
= & \pm 2 \sum_{s=1}^{(t p-1) / 2}\left(\frac{t p+1}{2}+s\right)\left(\begin{array}{c}
\lambda+1 \\
(q-1) / 2+s(q-1)
\end{array}\right)\left[v_{t p+1} x x\right] \\
& +\left[v_{t p+1} y x\right]+\left[v_{t p+1} x y\right] .
\end{aligned}
$$

This time the first $p$-adic digit of $(q-1) / 2+s(q-1)$ is the remainder of $-1 / 2-s$, so the values of $s$ for which the binomial coefficient does not vanish are $s \equiv(p-1) / 2$ $(\bmod p)$. But as in the previous case it is the coefficient

$$
\frac{t p+1}{2}+s \equiv \frac{t p+1}{2}+\frac{p-1}{2} \equiv \frac{t+1}{2} p \quad(\bmod p)
$$

that now vanishes, so that we are again left with (6b).

We now turn to $(6 \mathrm{c})$, which in this case requires us to prove that

$$
\left[v_{t p+1} y x\right]-\left[v_{t p+1} x x\right]
$$

is central. We have first

$$
0=\left[v_{t p+1}[y x y]\right]=-\left[v_{t p+1} y x y\right] .
$$

Also $\left[v_{t p+1} x x y\right]=0$; this is because

$$
\begin{aligned}
0= & {\left[v_{t p-1}\left[y x^{2 q-1} y\right]\right]=\left[v_{t p-1}\left[y x^{2 q-1}\right] y\right]-\left[v_{t p-1} y\left[y x^{2 q-1}\right]\right] } \\
= & {\left[v_{t p-1} y x^{2 q-1} y\right]+\left[v_{t p-1} x y x^{2 q-2} y\right]-(-1)^{2 q-3}\left(\begin{array}{c}
2 q-1 \\
2
\end{array}\right)\left[v_{t p-1} y x^{2 q-3} y x x\right] } \\
& -(-1)^{2 q-2}(2 q-1)\left[v_{t p-1} y x^{2 q-2} y x\right]-(-1)^{2 q-1}\left[v_{t p-1} y x^{2 q-1} y\right] .
\end{aligned}
$$

The first two terms cancel out, and so do the third and the fourth, so we get $\left[v_{t p+1} x x y\right]=$ 0 . So we are left with proving in this case

$$
\left[\left[v_{t p+1} y x\right]-\left[v_{t p+1} x x\right], x\right]=0 .
$$

Consider now

$$
\lambda=p(q-1)+1=q-2+(p-1)(q-1)+2 .
$$

so that $\lambda \equiv 1(\bmod p)$, and all other nonzero $p$-adic digits of $\lambda$ are $p-1$. Note also that

$$
\left[y x^{\lambda}\right]=\left[v_{p-1} x x\right],
$$

and $\left[y x^{\lambda} y\right]=0$, as $p>3$. We have

$$
0=\left[v_{(t-1) p+1}\left[y x^{\lambda} y\right]\right]=\left[v_{(t-1) p+1}\left[y x^{\lambda}\right] y\right]-\left[v_{(t-1) p+1} y\left[y x^{\lambda}\right]\right] .
$$


We compute first

$$
\begin{aligned}
{\left[v_{(t-1) p+1}\left[y x^{\lambda}\right]\right]=} & \sum_{s=0}^{p-1}\left(\left(\begin{array}{c}
\lambda \\
s(q-1)
\end{array}\right)\left[v_{(t-1) p+1+s} y x x^{\lambda-\cdots}\right]\right. \\
& \left.-\left(\begin{array}{c}
\lambda \\
s(q-1)+1
\end{array}\right)\left[v_{(t-1) p+1+s} x y x^{\lambda-\cdots}\right]\right)+\left[v_{t p+1} y x\right]-\left[v_{t p+1} x y\right] \\
= & \sum_{s=0}^{p-1}(s+1)\left(\begin{array}{c}
\lambda+1 \\
s(q-1)+1
\end{array}\right)\left[v_{t p+1} x x\right]+2\left[v_{t p+1} y x\right] .
\end{aligned}
$$

The first $p$-adic digit of $\lambda+1$ is 2 , whereas that of $s(q-1)+1$ is the remainder of $1-s(\bmod p)$. Thus the binomial coefficient vanishes except possibly for the values $s=0, s=1$ and $s=p-1$. In the last case the coefficient $s+1$ vanishes. The first two terms yield a total coefficient

$$
1\left(\begin{array}{c}
\lambda+1 \\
1
\end{array}\right)+2\left(\begin{array}{c}
\lambda+1 \\
q
\end{array}\right)=2-2=0
$$

Thus

$$
\left[v_{(t-1) p+1}\left[y x^{\lambda}\right] y\right]=2\left[v_{t p+1} y x y\right]=0,
$$

as we have seen. Now we compute

$$
\begin{aligned}
0=\left[v_{(t-1) p+1} y\left[y x^{\lambda}\right]\right]= & \sum_{s=1}^{p-1}\left(\begin{array}{c}
\lambda \\
s(q-1)-1
\end{array}\right)\left[v_{(t-1) p+1+s} y x x^{\lambda-\cdots}\right] \\
& \left.+\left(\begin{array}{c}
\lambda \\
s(q-1)
\end{array}\right)\left[v_{(t-1) p+1+s} x y x^{\lambda-\cdots}\right]\right)-\left(\begin{array}{l}
\lambda \\
2
\end{array}\right)\left[v_{t p+1} y x x\right] \\
& +\lambda\left[v_{t p+1} x y x\right]-\left[v_{t p+1} x x y\right] .
\end{aligned}
$$

We note first that the summation term equals

$$
\sum_{s=1}^{p-1}(s+1)\left(\begin{array}{c}
\lambda+1 \\
s(q-1)
\end{array}\right)\left[v_{t p+1} x \times x\right] .
$$

As above, the terms giving a non obviously zero contribution are $s=p-2$ and $s=p-1$. The last one yields a zero coefficient, whereas the first one yields a coefficient

$$
\begin{aligned}
\left(\begin{array}{c}
\lambda+1 \\
(p-2)(q-1)
\end{array}\right) & =\left(\begin{array}{l}
2+p(p-1)+p^{2}(p-1)+\cdots+(q / p)(p-1) \\
2+p(p-1)+p^{2}(p-1)+\cdots+(q / p)(p-3)
\end{array}\right) \\
& =\left(\begin{array}{c}
p-1 \\
p-3
\end{array}\right)=\left(\begin{array}{c}
p-1 \\
2
\end{array}\right)=1 .
\end{aligned}
$$


Of the final three terms, the last one has already been seen to vanish, and the first one has a zero coefficient

$$
\left(\begin{array}{l}
\lambda \\
2
\end{array}\right)=\frac{\lambda(\lambda-1)}{2}
$$

as $\lambda \equiv 1(\bmod p)$. We obtain

$$
\left[v_{t p+1} x x x\right]+\left[v_{t p+1} x y x\right]=0,
$$

as required.

\section{The easy case}

We finally deal with the case $i \not \equiv 0(\bmod p)$, which is considerably easier. The relation (6a) comes, via induction, from familiar arguments. The two expansions

$$
\begin{aligned}
0 & =\left[v_{i} x\left[y x^{j} y\right]\right]=\left[v_{i} x\left[y x^{j}\right] y\right]-\left[v_{i} x y\left[y x^{j}\right]\right] \\
& =\left[v_{i} x y x^{j} y\right]-(-1)^{j}\left[v_{i} x y x^{j} y\right]=-i\left(1-(-1)^{j}\right)\left[v_{i} x x x^{j} y\right],
\end{aligned}
$$

and

$$
\begin{aligned}
0 & =\left[v_{i}\left[y x^{j+1} y\right]\right]=\left[v_{i}\left[y x^{j+1}\right] y\right]-\left[v_{i} y\left[y x^{j+1}\right]\right] \\
& =\left[v_{i} y x x^{j} y\right]-(j+1)\left[v_{i} x y x^{j} y\right]-(-1)^{j+1}\left[v_{i} y x x^{j} y\right] \\
& =i\left(j+2+(-1)^{j}\right)\left[v_{i} x x x^{j} y\right]
\end{aligned}
$$

fail at the same time only when $j$ is even, and $j+3 \equiv 0(\bmod 3)$. In this case, we obtain $(6 a)$ by writing $j+3=\beta p^{t}$, with $\beta \not \equiv 0(\bmod p)$, and proceeding as we did to compute the length of the first chain. As before, we now easily prove (6d) as

$$
0=\left[\left[v_{i} x^{q-2}\right][y x y]\right]=-\left[v_{i+1} y y\right] .
$$

We obtain $(6 b)$ from

$$
\begin{aligned}
0= & {\left[v_{i}\left[y x^{q-1} y\right]\right]=\left[v_{i}\left[y x^{q-1}\right] y\right]-\left[v_{i} y\left[y x^{q-1}\right]\right] } \\
= & {\left[v_{i} y x x^{q-3} x y\right]-(q-1)\left[v_{i} x y x^{q-3} x y\right] } \\
& -(-1)^{q-2}(q-1)\left[v_{i} y x x^{q-3} y x\right]-(-1)^{q-1}\left[v_{i} y x x^{q-3} x y\right] \\
= & i\left((1-1-1)\left[v_{i+1} x y\right]-\left[v_{i+1} y x\right]\right) .
\end{aligned}
$$

We are left with proving $(6 \mathrm{c})$, that is,

$$
\left[v_{i+1} y x\right]=(i+1)\left[v_{i+1} x x\right],
$$


an identity in the even weight $(i+2)(q-1)+2$. We take

$$
\lambda=\frac{(i+2)(q-1)}{2} \equiv-\frac{i}{2}-1 \text {. }
$$

We need to calculate the expansion

$$
0=\left[\left[y x^{\lambda}\right]\left[y x^{\lambda}\right]\right]
$$

only to a small degree of accuracy. In fact, this gives

$$
0=\mu\left[v_{i+1} x x\right]-\lambda\left[v_{i+1} y x\right]+\left[v_{i+1} x y\right]=\mu\left[v_{i+1} x x\right]-(\lambda+1)\left[v_{i+1} y x\right],
$$

for some $\mu$. This shows that in $L$ there is a nontrivial linear relation between $\left[v_{i+1} x x\right]$ and $\left[v_{i+1} y x\right]$, as

$$
\lambda+1 \equiv-\frac{i}{2} \not \equiv 0 \quad(\bmod p) .
$$

But then we can read off this relation in the homomorphic image $N$ of $L$, where it is of course $(6 c)$.

\section{References}

[1] A. A. Albert and M. S. Frank, 'Simple Lie algebras of characteristic $p$ ', Univ. e Politec. Torino. Rend. Sem. Mat. 14 (1954-55), 117-139.

[2] M. Avitabile and G. Jurman, 'Diamonds in thin Lie algebras', Technical Report.

[3] R. Block, 'New simple Lie algebras of prime characteristic', Trans. Amer. Math. Soc. 89 (1958), $421-449$.

[4] R. Brandl, 'The Dilworth number of subgroup lattices', Arch. Math. (Basel) 50 (1988), 502-510.

[5] A. Caranti, 'Presenting the graded Lie algebra associated to the Nottingham group', J. Algebra 198 (1997), 266-289.

[6] A. Caranti and G. Jurman, 'Quotients of maximal class of thin Lie algebras. The odd characteristic case', Comm. Algebra (1999), to appear.

[7] A. Caranti and S. Mattarei, 'Gradings of non-graded Hamiltonian Lie algebras', Technical Report.

[8] A. Caranti, S. Mattarei and M. F. Newman, 'Graded Lie algebras of maximal class', Trans. Amer. Math. Soc. 349 (1997), 4021-4051.

[9] A. Caranti, S. Mattarei, M. F. Newman and C. M. Scoppola, 'Thin groups of prime-power order and thin Lie algebras', Quart. J. Math. Oxford Ser. (2) 47 (1996), 279-296.

[10] A. Caranti and M. F. Newman, 'Graded Lie algebras of maximal class. II', Technical Report.

[11] H. Garland, 'The arithmetic theory of loop groups', Inst. Hautes Études Sci. Publ. Math. 52 (1980), 5-136.

[12] G. Havas, M. F. Newman and E. A. O'Brien, 'ANU p-Quotient Program (version 1.4), written in $C$, available as a share library with GAP and as part of Magma, or from http: //wwwmaths . anu . edu . au/services/ftp.html', (School of Mathematical Sciences, Australian National University, Canberra, 1997). 
[13] B. Huppert, Endliche Gruppen. I, Die Grundlehren der mathematischen Wissenschaften, Band 134 (Springer, Berlin, 1967).

[14] N. Jacobson, Lie algebras, (Dover Publications Inc., New York, 1979, republication of the 1962 original).

[15] G. Jurman, 'Graded Lie algebras of maximal class. III', Technical Report.

[16] _ ' 'Quotients of maximal class of thin Lie algebras. The case of characteristic two', Comm. Algebra (1999), to appear.

[17] A. I. Kostrikin, 'The beginnings of modular Lie algebra theory', in: Group theory, algebra, and number theory (Saarbrücken, 1993) (de Gruyter, Berlin, 1996) pp. 13-52.

[18] C. R. Leedham-Green, 'The structure of finite p-groups', J. London Math. Soc. 50 (1994), 49-67.

[19] C. R. Leedham-Green and M. F. Newman, 'Space groups and groups of prime-power order. I', Arch. Math. (Basel) 35 (1980), 193-202.

[20] C. R. Leedham-Green, W. Plesken and G. Klaas, Pro-p-groups of finite width, Lecture Notes in Math. 1674 (Springer, Berlin, 1997).

[21] È. Lucas, 'Sur les congruences des nombres eulériens et des coefficients différentiels des fonctions trigonométriques, suivant un module premie', Bull. Soc. Math. France 6 (1878), 49-54.

[22] S. Mattarei, 'Some thin pro-p-groups', J. Algebra, to appear.

[23] B. H. Neumann, 'Some remarks on infinite groups', J. London Math. Soc. 12 (1937), 120-127.

[24] D. J. S. Robinson, A course in the theory of groups (Springer, New York, 1982).

[25] A. Shalev, 'The structure of finite p-groups: effective proof of the coclass conjectures', Invent. Math. 115 (1994), 315-345.

[26] A. Shalev and E. I. Zelmanov, 'Narrow Lie algebras I: a coclass theory and a characterization of the Witt algebra', J. Algebra 189 (1997), 294-331.

[27] H. Strade and R. Farnsteiner, Modular Lie algebras and their representations, Monographs Textbooks Pure Appl. Mathematics 166 (Marcel Dekker, New York, 1988).

\section{Dipartimento di Matematica}

Università degli Studi di Trento

via Sommarive 14

I-38050 Povo (Trento)

Italy

e-mail: caranti@science.unitn.it

e-mail: mattarei@science.unitn.it 